\title{
The Relativistic Time-Dependent Aharonov-Bohm Effect
}

\author{
Athanasios N. Petridis, Zachary Kertzman, Khinlay Win \\ Department of Physics and Astronomy, Drake University, Des Moines, USA \\ Email: athan.petridis@drake.edu
}

Received 28 July 2014; revised 23 August 2014; accepted 18 September 2014

Copyright (C) 2014 by authors and Scientific Research Publishing Inc.

This work is licensed under the Creative Commons Attribution International License (CC BY). http://creativecommons.org/licenses/by/4.0/

c) (i) Open Access

\section{Abstract \\ The relativistic Aharonov-Bohm (AB) effect is studied using the time-dependent Dirac equation. It is shown that if the initial electron distribution is a pulse it is possible to define time-dependent signals that distinguish the $A B$ effect from dipole and induced Coulomb interactions.}

\section{Keywords}

\section{Aharonov-Bohm Effect, Dirac Equation, Time-Dependent Quantum Mechanics}

\section{Introduction}

The Aharonov-Bohm (AB) effect [1], consisting of wave function phase shifts due to minimal coupling with four-vector potentials, observable by means of interference patterns, has been a cornerstone of gauge field theories. In its original magnetic version, electrons propagate around an impenetrable, very long solenoid, thus experiencing no Lorentz forces as the magnetic field is confined inside the solenoid. However, due to direct coupling with the magnetic potential outside the solenoid there is a phase shift producing an interference pattern. Specifically, the phase difference, $\Delta \delta$, introduced by a closed path $C$ encircling the solenoid in the vector field, $A$, is equal to the magnetic flux:

$$
\Delta \delta=\frac{\mathrm{e}}{\hbar} \oint_{C} \boldsymbol{A} \cdot \mathrm{d} \boldsymbol{r} .
$$

A similar effect arises from interactions with a scalar potential. There is experimental evidence for the existence of the AB effect [2] [3] and it has become stronger over the years [4]. Recently the existence of the effect has been demonstrated even during quantum tunneling [5].

The AB effect has its roots in the topology of the electromagnetic (EM) vacuum [6] which is not simplyconnected since the first homotopy group, $\pi_{1}$ of the compact, gauge group $U(1)_{\mathrm{EM}}$ is isomorphic to the group 
of integers $Z$. In the Standard-Model, however, the $U(1)_{\mathrm{EM}}$ subgroup is irregularly embedded into the $S U(2)_{L} \times U(1)_{Y}$ electro-weak symmetry group and it is non-compact leading to a trivial EM vacuum topology.

The plane-wave solution in the asymptotic limit has been obtained in the case electrons interacting with cosmic strings [7]. Also some theoretical models for dark matter predict the formation of dark cosmic strings that can interact with the Standard Model charged particles via the AB effect [8]. The AB effect is relevant to magnetic-field vortices in superconductors. One interesting result is macroscopic parity violation leading to a spontaneous Hall effect [9]. There has been substantial progress in mathematically solving the Schrödinger equation with wave-packets with $\mathrm{AB}$ interactions [10]. The effects of spin have been addressed in terms of the non-relativistic Pauli equation. Specifically the scattering amplitude has been obtained in this approximation [11].

There are questions, though, pertaining to the role of dipole-like fields due to the finite-longitudinal-size of physical solenoids and the influence of induced Coulomb charges in the conductors that make up the solenoids. Furthermore, a complete relativistic treatment of the problem can allow for the investigation of the very high energy range and, in this way, of the limits of applicability of the multiply-connected EM vacuum topology.

To this end, the AB effect is studied by means of the time-dependent relativistic Dirac equation with minimal EM coupling using a numerical algorithm. This equation automatically includes the effects of the electron spin. In this work, it is proposed that pulsed-beam experiments at high energy with very small-radius solenoids can disentangle the $\mathrm{AB}$ effect from competing dipole-field interactions and induced Coulomb charge potentials. This is within the reach of contemporary experiments. It is shown that asymmetrical induction signals can easily distinguish between solenoid, i.e., pure AB, and dipole effects and minimize the influence of induced Coulomb charges.

\section{Numerical Algorithm and the Dirac Equation}

The numerical algorithm to be employed in this work is the staggered-leap-frog method. It is applied on a spatial grid of bin-size $\Delta d$ with a time-step $\Delta t$. At every grid point, $\boldsymbol{r}$, the 4-dimentional spinor, $\Psi$, describing a spin-1/2 fermion, at time $t+2 \Delta t$ is computed using the spinor at time $t$ and the Hamiltonian, $\hat{H}$, operating on the spinor at time $t+\Delta t$ :

$$
\Psi(\boldsymbol{r}, t+2 \Delta t)=\Psi(\boldsymbol{r}, t)-2 i \Delta t \hat{H}(\boldsymbol{r}) \Psi(\boldsymbol{r}, t+\Delta t) .
$$

The spatial derivatives are calculated symmetrically to avoid false propagation of the spinor, biased in one direction. Totally reflecting boundary conditions are chosen to ensure that there is no loss in the probability density. Reflections off the grid boundaries are avoided by stopping the algorithm at their onset. Due to the relativistic covariance of the Dirac equation there are no superluminal single-energy components of the spinor so that possible interference of waves reflected off the grid boundaries with those in the region of interest are easily avoided. The method can be made as accurate as desired by choosing a small spatial grid bin. However, stability may be an issue.

The Dirac equation is ( $c=\hbar=1$ and $\alpha_{j}, \quad \beta$ are $4 \times 4$ Dirac matrices, $j=1,2,3$ )

$$
i \partial_{t} \Psi(\boldsymbol{r}, t)=\hat{H} \Psi(\boldsymbol{r}, t), \quad \hat{H}=\boldsymbol{\alpha} \cdot \hat{\boldsymbol{\pi}}+\beta m+V(\boldsymbol{r}),
$$

where $V$ is a scalar potential and the canonical momentum operator is given by the minimal coupling scheme as

$$
\hat{\boldsymbol{\pi}}=\hat{\boldsymbol{p}}-\mathrm{e} \boldsymbol{A},
$$

where $A_{j}(j=1,2,3)$ are the vector potential components and e the electric charge of the fermion. A simple way to explore the region of stability of a numerical algorithm solving a differential equation is to use a plane wave trial function (Von-Neuman criterion). This method applied to the largest component of the spinor yields for the free Dirac equation

$$
\Delta d^{4}\left(1-(\Delta t)^{2}\right)-2(\Delta d \Delta t)^{2}-4(\Delta t)^{2}>0 .
$$

Since the equation is usually coupled, a numerical way to safeguard stability is needed. To this end, evaluating the norm of the spinor at each time step and checking for deviations from unity proved very effective. When instability ensues the norm starts deviating from unity very rapidly and/or it oscillates wildly. Stability is achieved by selecting an appropriately small time step. Since the algorithm requires information on the spinor from two previous time-steps, the first step is taken using a simple linear approximation. To further increase sta- 
bility, a predictor-corrector step is inserted but proved unnecessary in the cases presented here.

In order to calculate the time dependence of the probability distributions, the initial spinor is chosen to be a wave packet with group momentum $p_{0 j}$ and initial center location $r_{0 j}(j=1,2,3)$. Its probability density, $\rho=\Psi^{\dagger} \Psi$, is Gaussian in space with standard deviation $\sigma_{0}$,

$$
\Psi(\boldsymbol{r}, 0)=N \sqrt{\frac{E+m}{2 m}}\left[\begin{array}{c}
1 \\
0 \\
0 \\
\frac{p_{0}}{E+m}
\end{array}\right] \operatorname{Exp}\left(i \boldsymbol{p}_{0} \cdot\left(\boldsymbol{r}-\boldsymbol{r}_{0}\right)-\frac{\left(r-r_{0}\right)^{2}}{4 \sigma_{0}^{2}}\right), \quad E=\sqrt{p_{0}^{2}+m^{2}},
$$

where $N$ is an overall normalization factor and the spinor structure is in the matrix. When $\sigma_{0} \rightarrow \infty$, $\Psi$ becomes a positive energy plane wave in 1 dimension or a circular wave in 2 dimensions, which, for $p_{0}=0$, is a spin $+1 / 2$ eigenfunction.

\section{One-Dimensional Test Studies}

Studies of the numerical solutions to the time-dependent Dirac equation in 1 spatial dimension are a necessary step to affirm the validity, accuracy and stability of the method and compare with results obtained by other authors. The simplest example is, of course, that of a freely propagating fermion. It is known that high-frequency (approximately $2 \mathrm{E}$ ) oscillations, Zitterbewegung (jitter), occur in many observables, including the expectation value and the standard deviation of the position and the expectation value of the component of the spin in the direction perpendicular to the propagation. The expectation value of the position, $\langle x\rangle$, is shown in Figure 1 for various values of $p_{0}$ and $\sigma_{0}$ after subtraction of the overall drift. For fixed $p_{0}$ the jitter has a non-linear dependence on $\sigma_{0}$ and is maximized when $2 \sigma_{0}=\lambda_{C}$ (the Compton wavelength with $m=1$ ). It increases with $p_{0}$ but decays away with time.

In Figure 2 the standard deviation, $\sigma$, is plotted versus time for various values of $p_{0}$ and $\sigma_{0}$. A narrower or

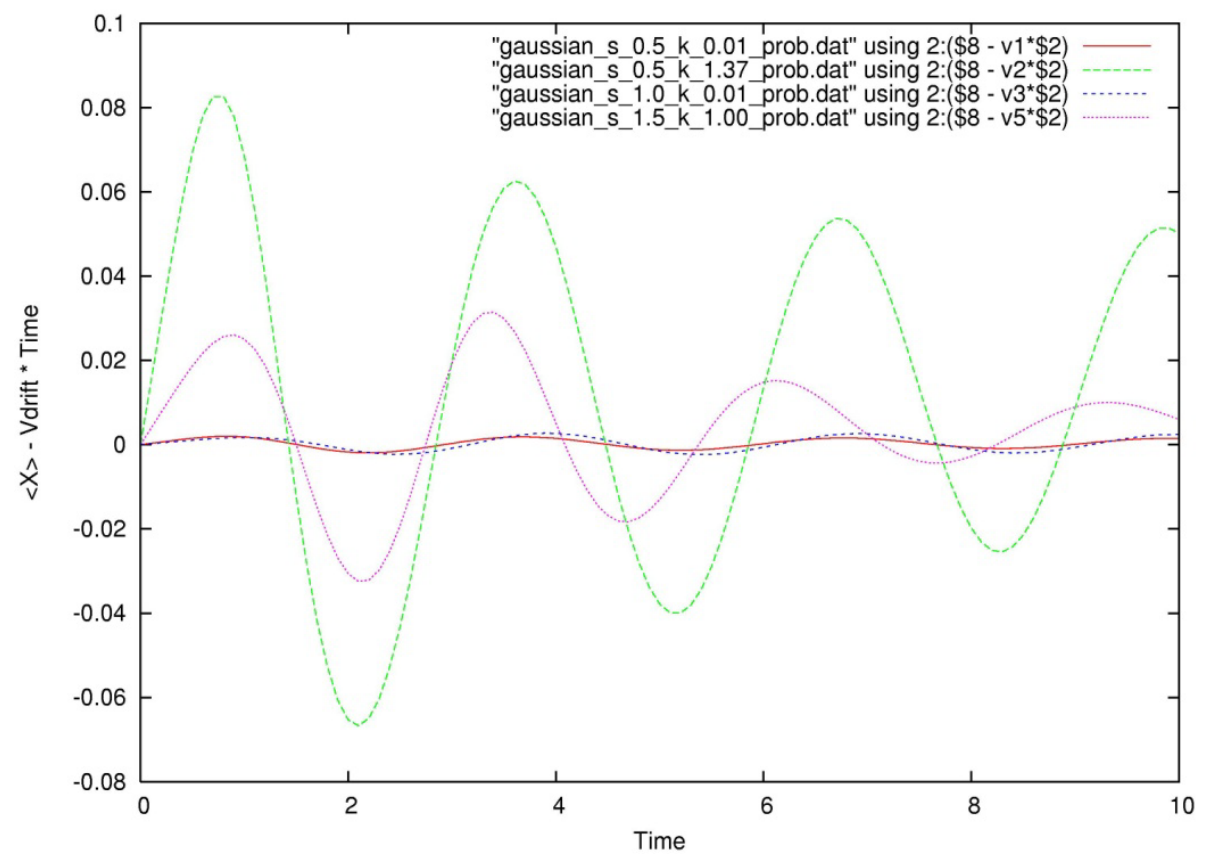

Figure 1. The position expectation value, $\langle x\rangle$, versus time after subtraction of the drift (red: $\sigma_{0}=0.5, p_{0}=0.01$; green: $\sigma_{0}=0.5, p_{0}=1.37$; blue: $\sigma_{0}=1.0, p_{0}=0.01$; purple: $\sigma_{0}=1.5, p_{0}=$ 0.01 ). The time-decaying jitter is apparent. For fixed $p_{0}$, it is maximal when the spread of the probability density, $2 \sigma_{0}$, is equal to the Compton wavelength, $\lambda_{C}$. It becomes more pronounced as $p_{0}$ increases. 


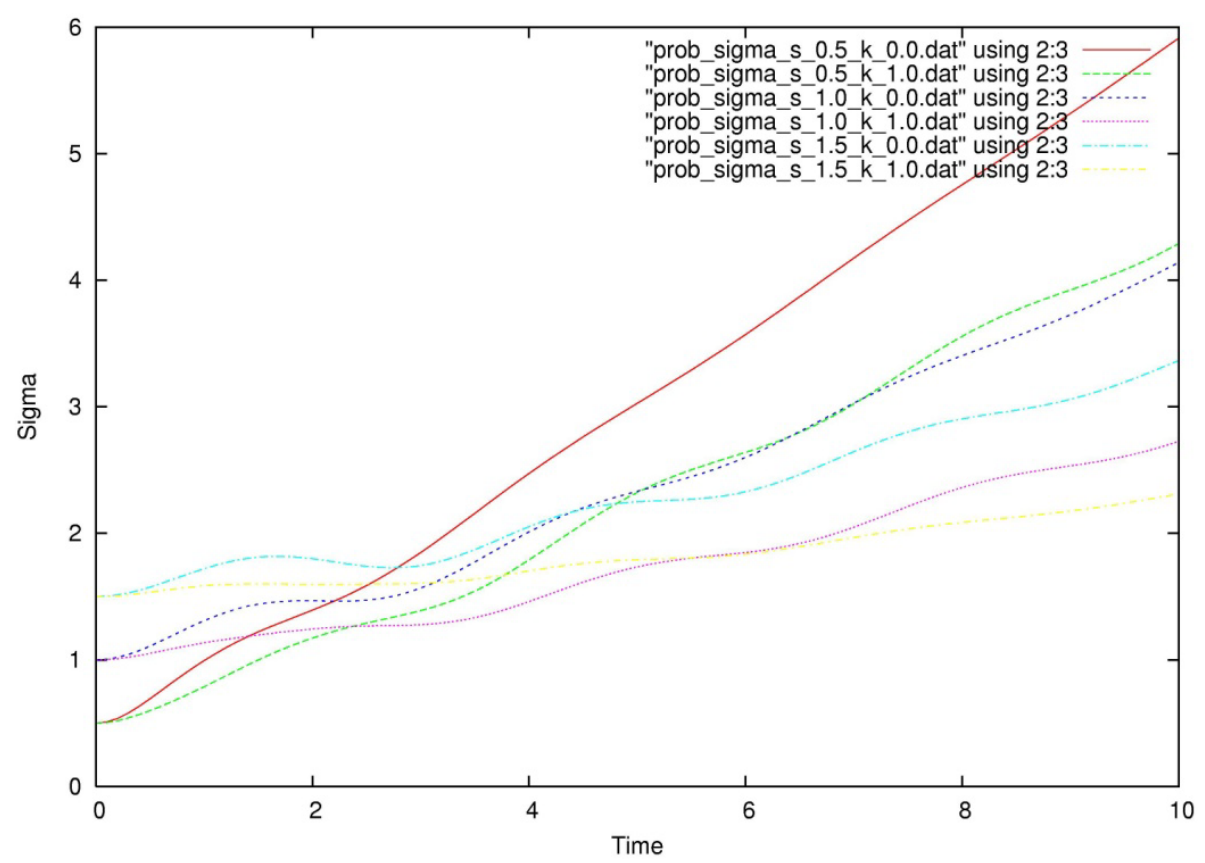

Figure 2. The position standard deviation, $\sigma$, versus time (red: $\sigma_{0}=0.5, p_{0}=0$; green: $\sigma_{0}=$ 0.5, $p_{0}=1.0$; blue: $\sigma_{0}=1.0, p_{0}=0$; purple: $\sigma_{0}=1.0, p_{0}=1.0$; light blue: $\sigma_{0}=1.5, p_{0}=0$; yellow: $\left.\sigma_{0}=1.5, p_{0}=1.0\right)$. An initially narrower or slower wave packet spreads out faster with time. The jitter is more pronounced at $2 \sigma_{0}=\lambda_{C}$ and dies out with time.

slower initial wave packet spreads out faster with time. The jitter decays away with time and is more pronounced at $2 \sigma_{0}=\lambda_{C}$. In Figure 3 the expectation value of the spin-component perpendicular to the propagation direction,

$$
\left\langle S_{z}\right\rangle=1 / 2 \int\left(\left|\Psi^{(0)}\right|^{2}-\left|\Psi^{(1)}\right|^{2}+\left|\Psi^{(2)}\right|^{2}-\left|\Psi^{(3)}\right|^{2}\right) \mathrm{d} x
$$

is plotted versus time where, $\Psi^{(\mu)}(\mu=0,1,2,3)$ are the 4 components of the spinor, $x(\mu=1)$ is the propagation direction and $z(\mu=3)$ is the direction perpendicular to it. It should be noted that this observable depends on the initial group momentum and that because the initial spinor is not a plane wave the jitter persists even when the initial group momentum is 0 . It decays away with time and is maximal at $2 \sigma_{0}=\lambda_{C}$. The results for $\langle x\rangle$ and $\left\langle S_{z}\right\rangle$ are in complete agreement with those produced by J. W. Braun, Q.

Su, and R. Grobe [12], who applied a split-operator technique to solve the Dirac equation employing a supercomputer. The results shown here were obtained using only a small computer.

\section{Infinite-Solenoid and Dipole Fields}

In this section numerical results on the time-dependent relativistic $\mathrm{AB}$ effect are presented. The Dirac equation describing a Gaussian-shaped electron pulse is coupled with a scalar and a vector potential. The propagation happens in the $x-y$ plane. An infinitely long solenoid of radius $R$ is placed perpendicularly to it. The scalar potential $V$ is used to define the wall of the solenoid. To achieve this, a very large, positive, constant value is given to $V$ inside the cylinder; it is kept at 0 outside. Quantum tunneling into and through the cylinder is almost entirely eliminated. The vector potential produced by an infinitely long solenoid centered at the origin is oriented azimuthally,

$$
A_{\mathrm{sol}}=\frac{A_{0}}{r} \hat{\phi}, r \geq R \text { and } \boldsymbol{A}_{\mathrm{sol}}=\frac{A_{0}}{R^{2}} r \hat{\phi}, r \leq R,
$$

where $r$ is the distance from the center and $A_{0}$ is the potential strength (it depends linearly on the current in the solenoid). If the solenoid is finite in length a dipole component is also generated. It is assumed that small magnetic 


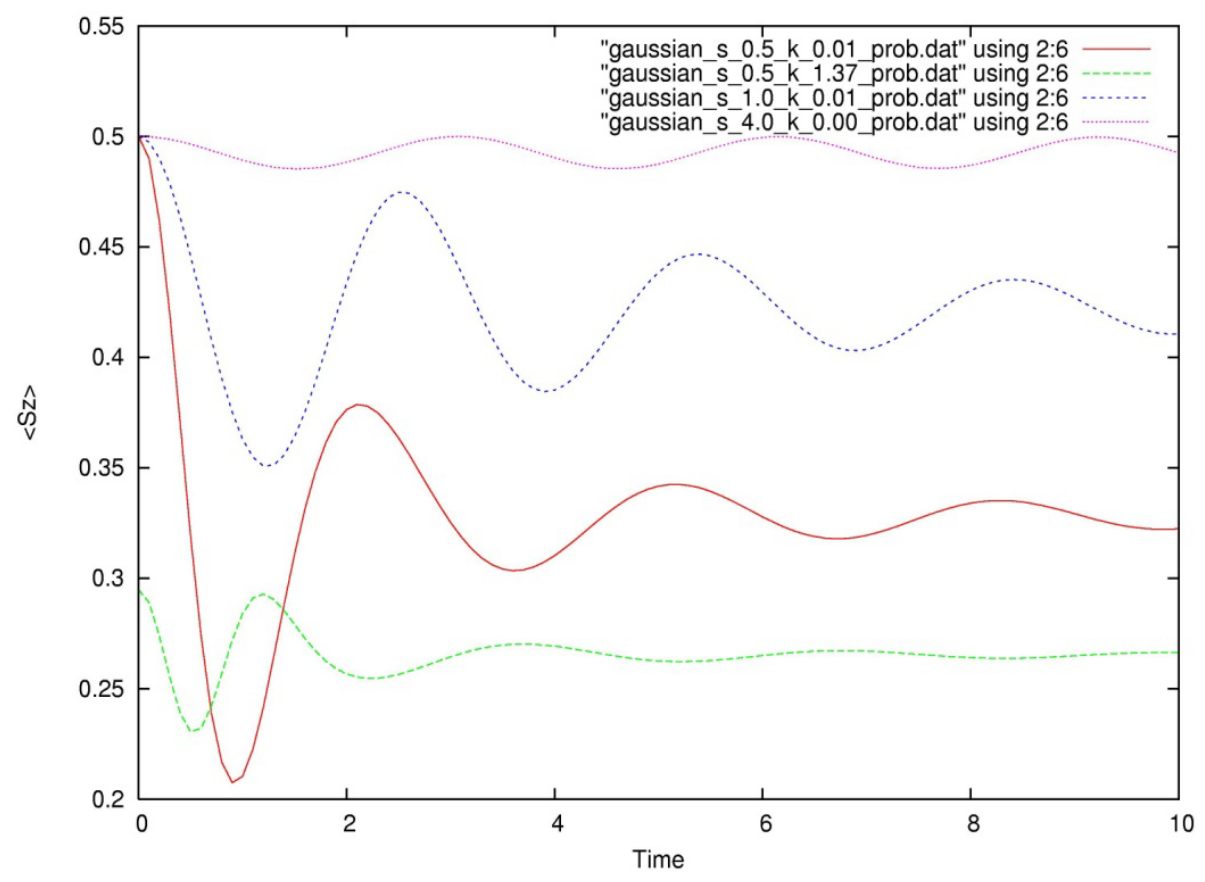

Figure 3. The expectation value of the spin component perpendicular to the propagation direction versus time (red: $\sigma_{0}=0.5, p_{0}=0.01$; green: $\sigma_{0}=0.5, p_{0}=1.37$; blue: $\sigma_{0}=1.0, p_{0}=$ 0.01 ; purple: $\sigma_{0}=4.0, p_{0}=0.0$ ). The jitter is present even when $p_{0}=0.0$, is more pronounced at $2 \sigma_{0}=\lambda_{C}$ and dies out with time.

fields produced around individual wires of the solenoid can be neglected due to the presence of the effectively impenetrable, smooth wall around the structure. The vector potential due to a simple, ideal dipole placed perpendicularly to the $\mathrm{x}-\mathrm{y}$ plane at the origin is

$$
A_{\text {dip }}=\frac{B_{0}}{r^{2}} \hat{\phi}
$$

where $B_{0}$ is its strength. Unlike the solenoid potential this corresponds to a non-zero magnetic field for $r>R$. In the calculations to be presented in this article the initial wave packet has group momentum $p_{0}=1.134$ with initial standard deviation $\sigma_{0}=5$. It travels diagonally across the $x-y$ plane starting from the lower-left corner. The solenoid is centered at $x=y=0$ and has radius $R=4$.

First, a pure solenoid field is examined with $A_{0}=0.5$. In Figure 4 a time sequence of the probability density distribution is presented. The asymmetry of the diffraction pattern around the solenoid is very obvious. At large times the distribution is disrupted. In Figure 5 a time sequence of the probability density distribution is presented for a pure dipole field with $B_{0}=0.5$. The asymmetry of the diffraction pattern around the solenoid is obvious as well. Since the dipole field falls-off more rapidly than the solenoid one the asymmetry is substantially smaller. In both cases some part of the probability density is reflected backwards and some part wraps around the solenoid due to the coupling with the vector potential. In all computations shown the spatial grid bin-size is $\Delta d=0.5$ and the time step is $\Delta t=0.0002$. In Figure 4 and Figure 5 the time sequence is left to right and, then, upper to lower panel corresponding to $t=12,24,36,48,60,72,84,96,108,120$.

\section{Characteristic Signals}

The time-dependent asymmetry of the diffraction pattern can be exploited in order to identify the type of potential that produces it. Since the effect is typically measured by inductively-coupled devices, sensitive to the local current density fluctuations and placed around the solenoid, a good measure would be the difference between the integrated densities left and right of the initial propagation direction. Specifically the probability density can be separately integrated in the upper-left and the lower-right quadrants (Figure 4 and Figure 5) and the (Left-Right) signal (a function of time) can be defined as 

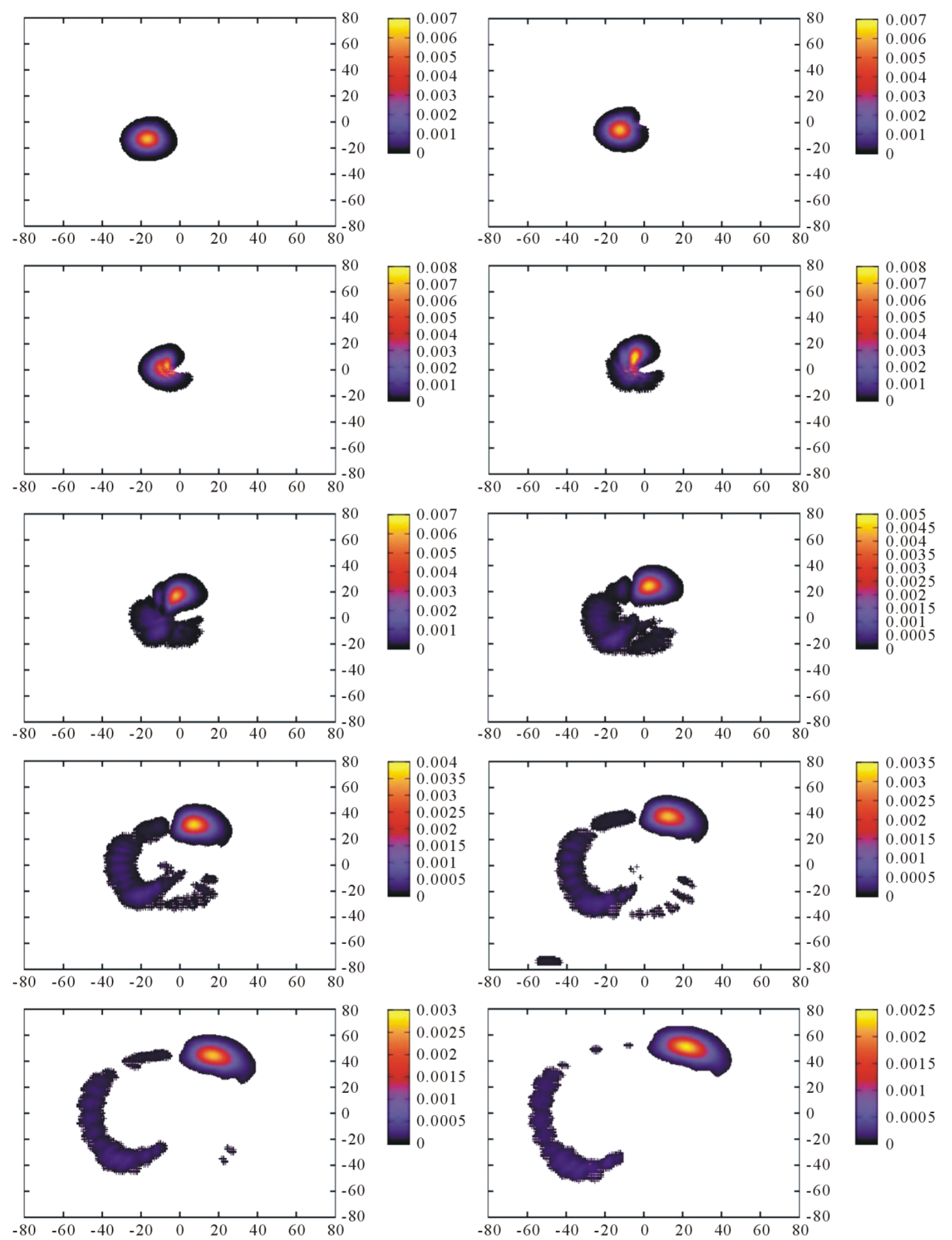

Figure 4. Probability density distribution for diffraction off a solenoid field with strength $A_{0}=0.5$. The initial spinor has $p_{0}$ $=1.134$ and $\sigma_{0}=5$ and moves diagonally. The solenoid is at the center and has radius $R=4$. The time sequence is left to right and, then, upper to lower panel: $t=12,24,36,48,60,72,84,96,108,120$. 

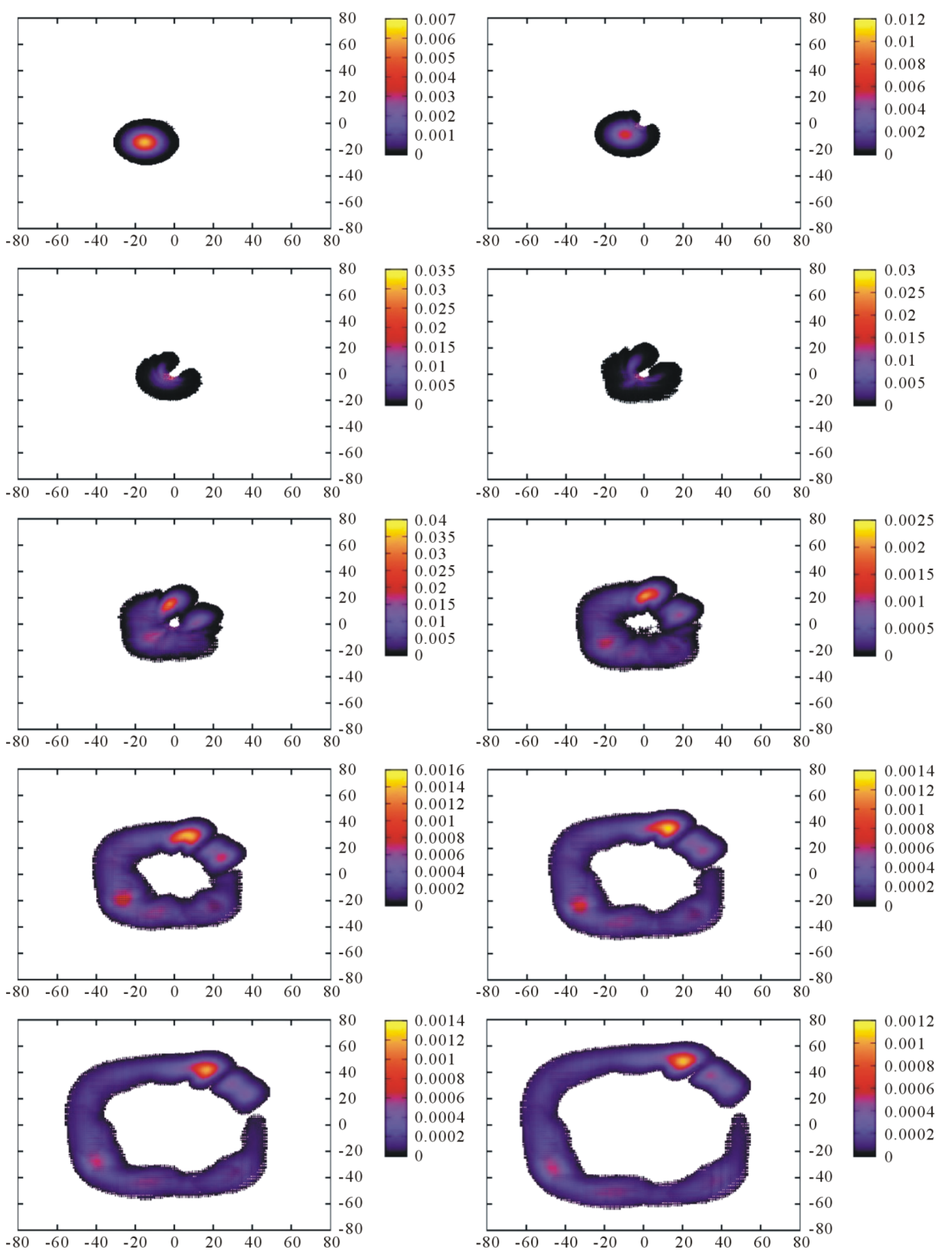

Figure 5. Probability density distribution for diffraction off a dipole field with strength $B_{0}=0.5$. The initial spinor has $p_{0}=$ 1.134 and $\sigma_{0}=5$ and moves diagonally. The solenoid is at the center and has radius $R=4$. The time sequence is left to right and, then, upper to lower panel: $t=12,24,36,48,60,72,84,96,108,120$. 


$$
S(t)=\iint_{\text {Upper-left }} \rho(x, y) \mathrm{d} x \mathrm{~d} y-\iint_{\text {Lower-right }} \rho(x, y) \mathrm{d} x \mathrm{~d} y .
$$

The signal obtained with a pure solenoid field is presented in Figure 6 as a function of time for increasing values of the potential strength $A_{0}$, indicated beside each curve. The signal has the form of a pulse that becomes broader as $A_{0}$ increases. It is important to note that the signal-peak dependence on $A_{0}$ is non-monotonic. It is maximized around $A_{0}=1$. This behavior can be understood as follows. As the field strength increases a point is reached at which the probability density is swung around the solenoid resulting in a reduced difference between the upper-left and lower-right quadrant integrals.

The signal obtained with a pure dipole field is presented in Figure 7 as a function of time for increasing values of the potential strength $B_{0}$, indicated beside each curve. The signal has the form of a pulse that becomes broader as $B_{0}$ increases. In the range of potential strengths investigated, the peak signal is monotonic in $B_{0}$. In this range the effect of the probability density swinging around the solenoid is not as large as in the case of the pure solenoid field because the dipole field dies out faster with the distance from the center.

For comparison the signal for a solenoid field of strength $A_{0}$ superimposed with a dipole field of strength $B_{0}=0.1 \cdot A_{0}$ is shown in Figure 8. Similarly the signal for a dipole field superimposed with a solenoid field of strength $A_{0}=0.1 \cdot B_{0}$ is shown in Figure 9. In both figures the curves are marked with the total strength $A_{0}+B_{0}$. It is clear that the behavior follows that of the dominant field. The exact form of the curves can allow for the determination of whether there is diffraction due to a pure solenoid field (the essence of the AB effect) in a given experimental setting.

The results obtained can be further elucidated by examining the dependence of the peak signal, i.e., the maximal value of the Left-Right signal, on the total field strength, $A_{0}+B_{0}$ (Figure 10). When the solenoid field dominates the non-monotonic behavior is clear. Another observable that provides a very clear distinction between solenoid and dipole fields is the time at which the Left-Right signal peak occurs. This is plotted in Figure 11 versus the total field strength. In the case of solenoid-dominated fields a maximum appears at intermediate values of $A_{0}+B_{0}$.

A pulsed experiment may, therefore, be designed. An oscilloscope can easily produce the Left-Right signal captured by induction detectors. The experiment can be repeated for various field strengths and the signal shapes

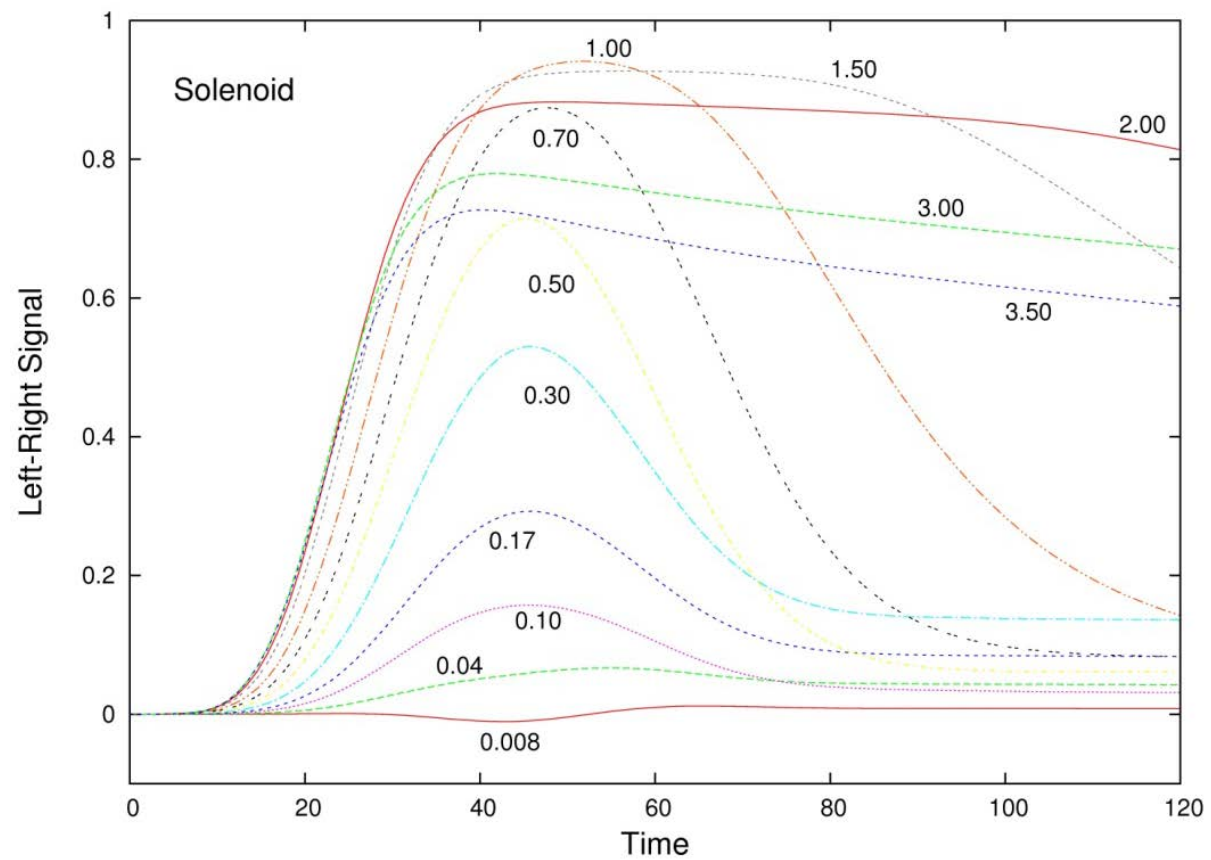

Figure 6. Left-Right signal versus time for increasing values of a pure solenoid field whose strength, $A_{0}$, is indicated beside each curve. The pulse becomes broader as $A_{0}$ increases. The peak value is a non-monotonic function of $A_{0}$. 


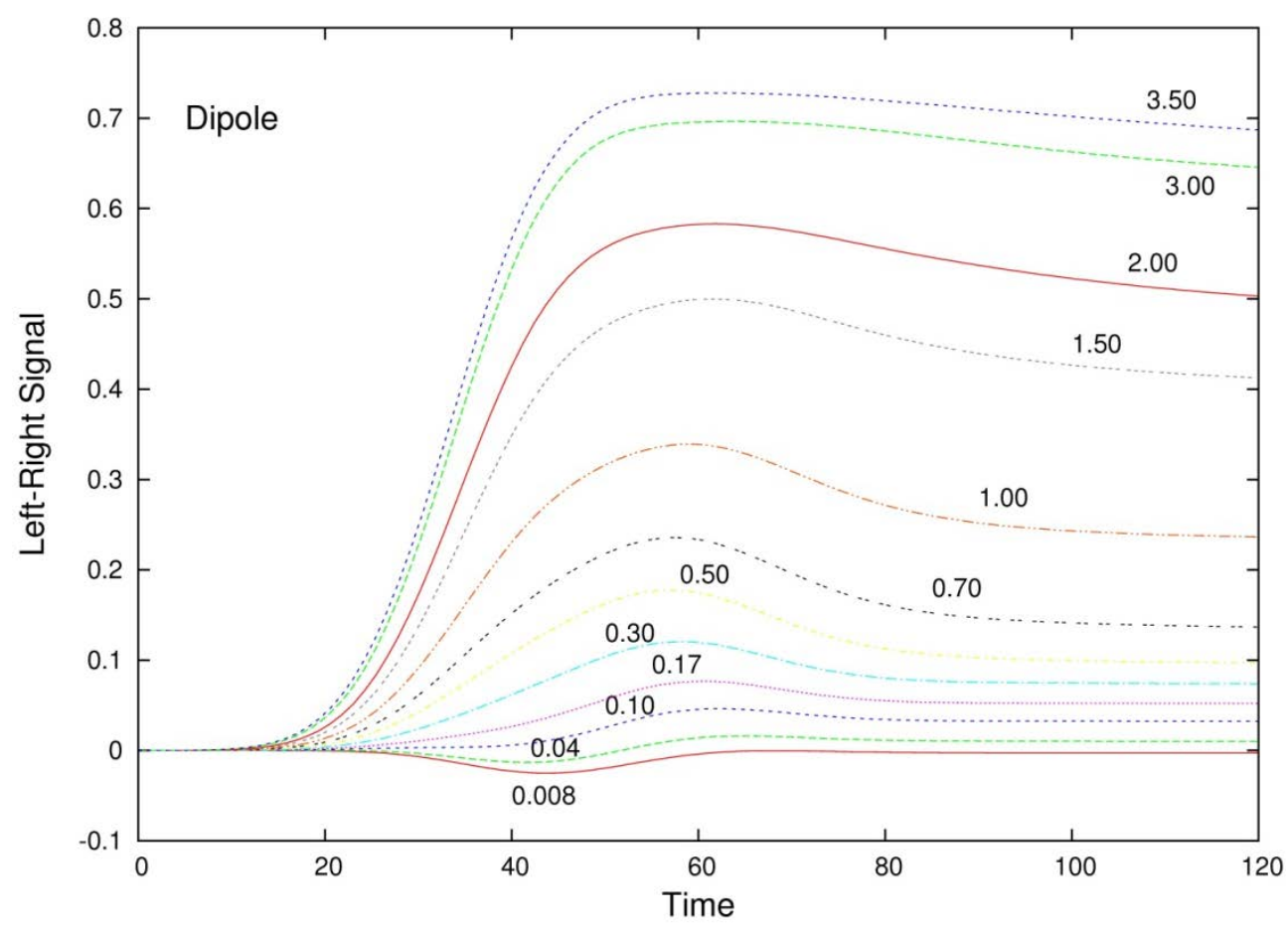

Figure 7. Left-Right signal versus time for increasing values of a pure dipole field whose strength, $B_{0}$, is indicated beside each curve. The pulse becomes broader as $B_{0}$ increases. In this field-strength range, the peak value is a monotonic function of $B_{0}$.

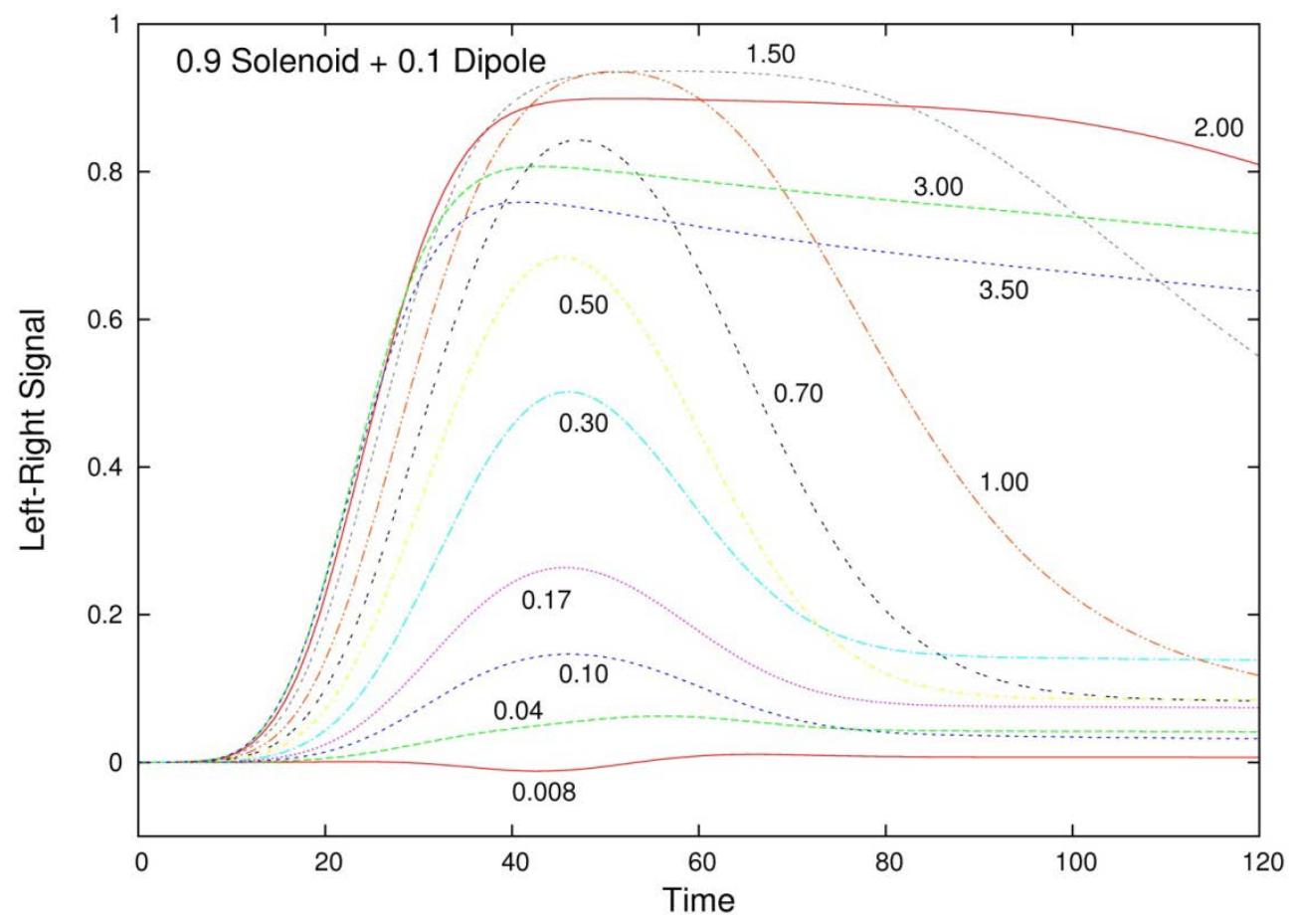

Figure 8. Left-Right signal versus time for increasing values of a 0.91 solenoid +0.09 dipole field whose total strength is indicated beside each curve. The pulse becomes broader as the field increases. The peak value is a non-monotonic function of the field strength. The overall behavior is solenoidlike. 


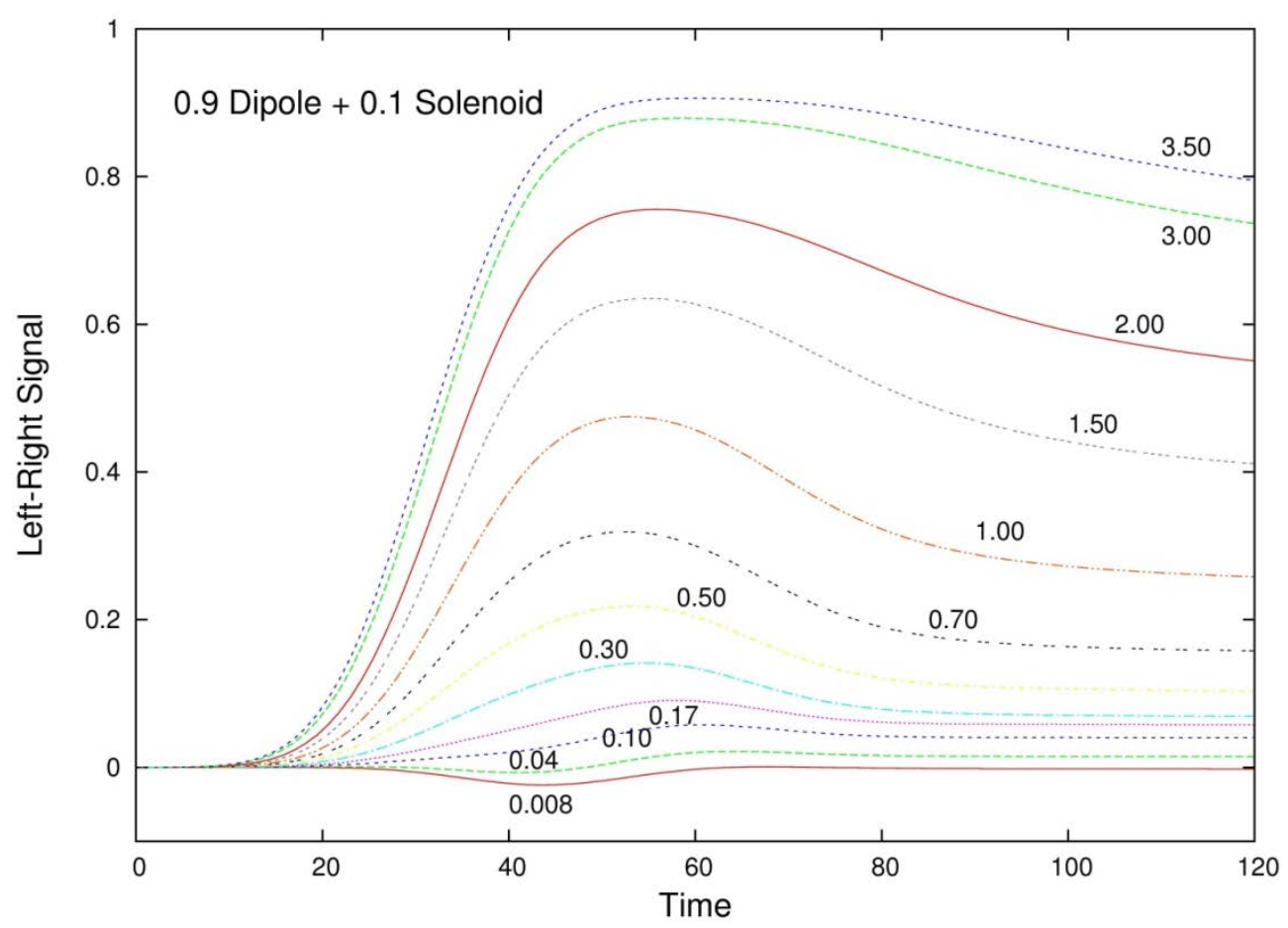

Figure 9. Left-Right signal versus time for increasing values of a 0.91 dipole +0.09 solenoid field whose total strength is indicated beside each curve. The pulse becomes broader as the field increases. The peak value is a monotonic function of the field strength in this range. The overall behavior is dipole-like.

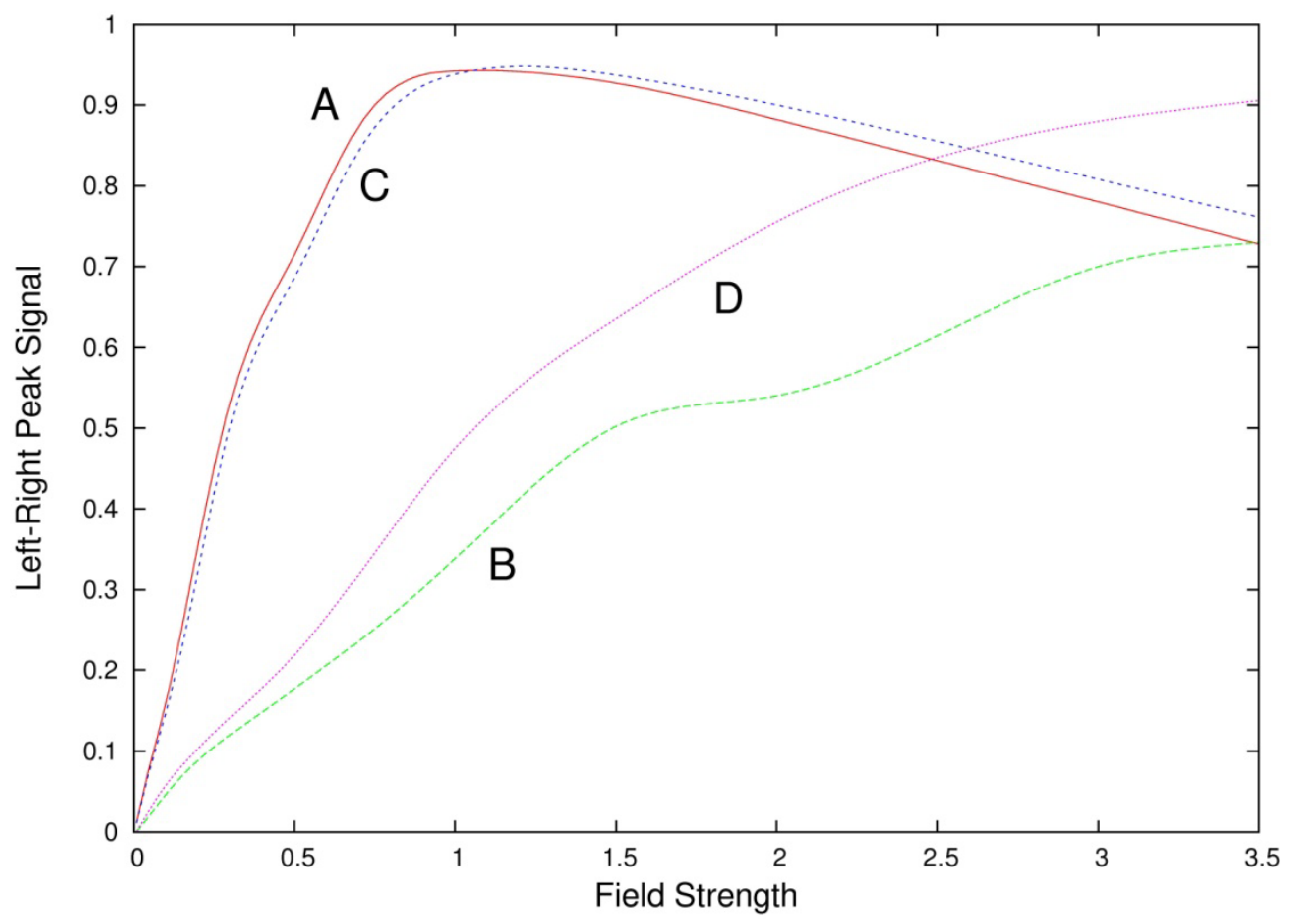

Figure 10. Left-Right signal peak value versus the total field strength, $A_{0}+B_{0}$. A: solenoid, B: dipole, C: 0.91 solenoid +0.09 dipole, $\mathrm{D}: 0.91$ dipole +0.09 solenoid. The behavior distinctly shows the presence of the solenoid field contribution. 


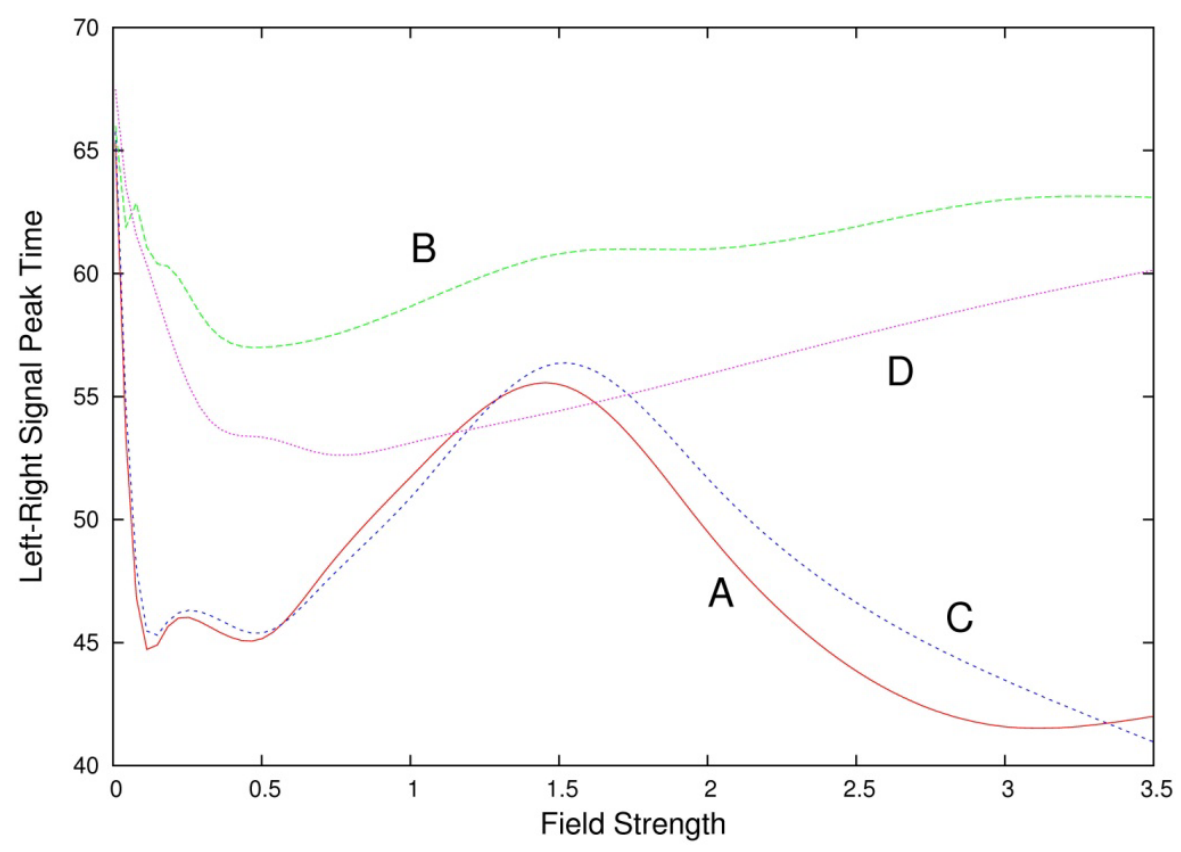

Figure 11. Left-Right signal peak time versus the total field strength, $A_{0}+B_{0}$. A: solenoid, B: dipole, C: 0.91 solenoid +0.09 dipole, $\mathrm{D}$ : 0.91 dipole +0.09 solenoid. The maximum at intermediate field strengths (curves A and C) distinctly shows the presence of the solenoid field contribution.

versus time and field strength can confirm the existence of the $A B$ effect even in the presence of a residual dipole field.

\section{Induced Coulomb Charges}

The initial physical size of the electron pulses described in the previous section has been chosen to have $\sigma_{0}=5$. Given that $\lambda_{C}=2.4263 \times 10^{-12} \mathrm{~m}$, for electrons, the solenoid radius is $R=4=1.55 \times 10^{-12} \mathrm{~m}$ (one unit of length corresponds to $3.87 \times 10^{-13} \mathrm{~m}$ ). For a solenoid-dominated field with $A_{0} \leq 1.0$ the left-right signal rise time is about 40 to 50 time units, i.e., $5.15 \times 10^{-21} \mathrm{~s}$ to $6.44 \times 10^{-21} \mathrm{~s}$ (one time unit corresponds to $1.288 \times 10^{-22} \mathrm{~s}$ ) and the signal duration is in the range of 60 to 120 time units, i.e., $7.73 \times 10^{-21} \mathrm{~s}$ to $1.55 \times 10^{-20} \mathrm{~s}$. An actual experiment can be scaled up to several orders of magnitude larger physical dimensions. However, there is a certain advantage in keeping the pulses and the solenoid as small as possible. It allows for easier discrimination against the effect of induced Coulomb charges on the solenoid conductor. These can influence the motion of the electron beam by Lorenz forces, unrelated to the AB effect.

It is known [13] [14] that the time-development of induced charges and the establishment of potentials between them and the outside (inducing) charges placed or moving in front of conductors involve phenomena at the atto-second $\left(10^{-18} \mathrm{~s}\right)$ up to femto-second $\left(10^{-15} \mathrm{~s}\right)$ scales. The details depend on the intrinsic plasma frequencies of conduction electrons in the specific material. The induced Coulomb potentials oscillate about their "infinite-time" (static) values with periods of several atto-seconds and converge to them in femto-seconds. The convergence is faster and the amplitude of those oscillations is smaller when the outside charges are closer to the surface of the conductor. Thus, fast moving pulses of electrons approaching small solenoids may produce $\mathrm{AB}$ effect signals that develop at time scales that are shorter than the induced potential formation times, minimizing the effect of the latter on the measurements. A simple extrapolation of the results obtained in the previous section shows that for a weak $\left(A_{0} \leq 1.0\right)$ magnetic field confined to a radius of $10^{-8} \mathrm{~m}$ the AB effect signal will have a duration of about $10^{-16} \mathrm{~s}$. This is shorter than the times needed to establish an induced potential.

\section{Conclusion}

The time-development of the relativistic AB effect has been investigated using the Dirac equation with minimal 
coupling. Comparisons have been made with dipole-field effects. A method using pulsed electron beams is proposed to disentangle the $\mathrm{AB}$ effect from the influence of residual dipole fields, present due to the finite size of solenoids. The proposed Left-Right signals exhibit a distinctively different time-development when solenoid or dipole-dominated fields are present and their dependence of the field strength is characteristic of the type of field. Furthermore, this method can help minimize the effects of induced charges in the conductor. The time-dependent approach can be used to study the $\mathrm{AB}$ effect and investigate its behavior at relativistic energies.

\section{Acknowledgements}

Z. K. and K.W. have been partially supported through summer internships by the Drake Undergraduate Science Collaborative Institute (DUSCI).

\section{References}

[1] Aharonov, Y. and Bohm, D. (1959) Physical Review, 115, 485-491. http://dx.doi.org/10.1103/PhysRev.115.485

[2] Tonomura, A., et al. (1982) Physical Review Letters, 48, 1443-1446. http://dx.doi.org/10.1103/PhysRevLett.48.1443

[3] Tonomura, A., et al. (1986) Physical Review Letters, 56, 792-795. http://dx.doi.org/10.1103/PhysRevLett.56.792

[4] Caprez, A.P., et al. (2007) Physical Review Letters, 99, Article ID: 210401 (4).

[5] Noguchi, A., et al. (2014) Nature Communications, 5, Article No. 3868.

[6] Ryder, L.H. (1996) Quantum Field Theory. Cambridge University Press, Cambridge. http://dx.doi.org/10.1017/CBO9780511813900

[7] Alford, M.G. and Wilczek, F. (1989) Physical Review Letters, 62, 1071-1074. http://dx.doi.org/10.1103/PhysRevLett.62.1071

[8] Vachaspati, T. (2009) Physical Review D, 80, Article ID: 063502 (5).

[9] Emparan, R. and Valle Basagoti, M.A. (1994) Physical Review B, 49, 14460-14465. http://dx.doi.org/10.1103/PhysRevB.49.14460

[10] Ballesteros, M. and Weder, R. (2009) Journal of Mathematical Physics, 50, 122108-122162. http://dx.doi.org/10.1063/1.3266176

[11] Khalikov, V.R. and Ho, C.-L. (2008) Annals of Physics, 323, 1280-1293. http://dx.doi.org/10.1016/j.aop.2007.08.007

[12] Brawn, J.W., Su, Q. and Grobe, R. (1999) Physical Review A, 59, 604-612. http://dx.doi.org/10.1103/PhysRevA.59.604

[13] Alducin, M., Díez Muiño, R. and Juaristi, J.I. (2003) Journal of Electron Spectroscopy and Related Phenomena, 129, 105-109. http://dx.doi.org/10.1016/S0368-2048(03)00057-4

[14] Díez Muiño, R., Sánchez-Portal, D., Silkin, V.M., Chulkov, E.V. and Echenique, P.M. (2011) Proceedings of the National Academy of Sciences of the USA, 108, 971-976. 
Scientific Research Publishing (SCIRP) is one of the largest Open Access journal publishers. It is currently publishing more than 200 open access, online, peer-reviewed journals covering a wide range of academic disciplines. SCIRP serves the worldwide academic communities and contributes to the progress and application of science with its publication.

Other selected journals from SCIRP are listed as below. Submit your manuscript to us via either submit@scirp.org or Online Submission Portal.
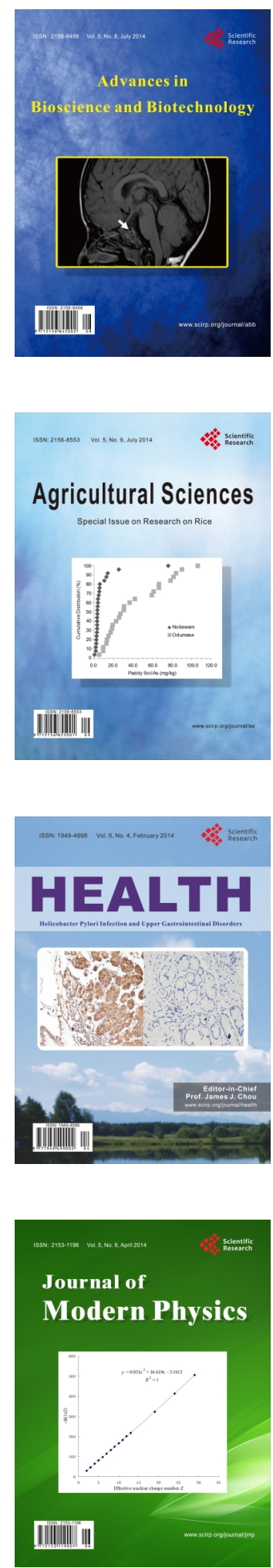
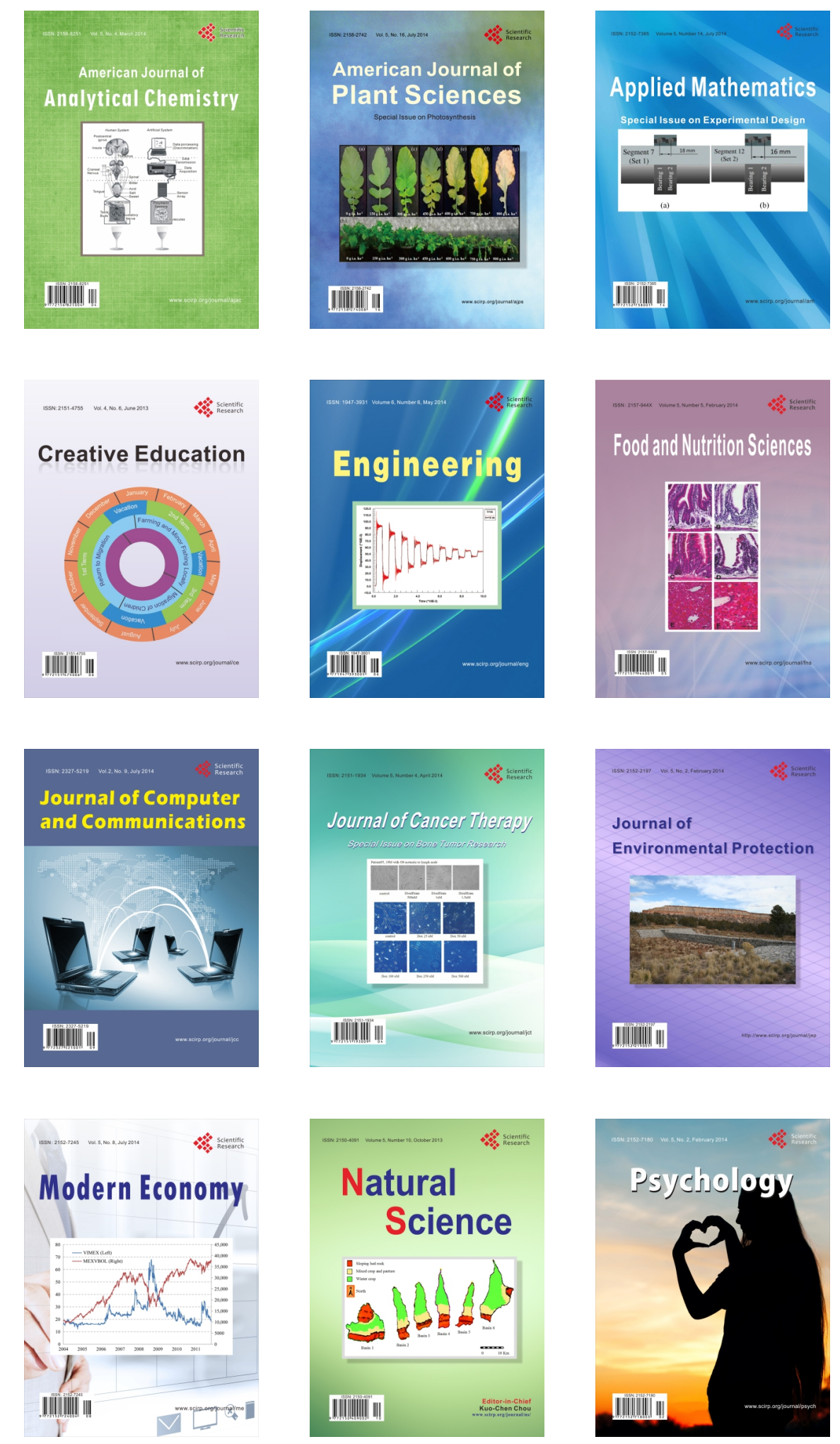